\title{
Multimode quantum analysis of an interferometer with moving mirrors
}

\author{
A. Luis and L.L. Sánchez-Soto \\ Departamento de Optica, Facultad de Ciencias Físicas, Universidad Complutense, 28040 Madrid, Spain
}

(Received 31 January 1992)

\begin{abstract}
A multimode quantum analysis of noise reduction in interferometric measurements is presented. A unitary transformation relating the output modes with the input ones for a Michelson interferometer with moving mirrors is given. The evaluation of the noise spectrum shows that with a proper choice of the input states, the sensitivity of the interferometer can be pushed beyond the standard quantum limit. The results are compared with a semiclassical analysis.

PACS number(s): 42.50.Dv, 42.50.Lc, 03.65.Bz, 07.60.Ly
\end{abstract}

\section{INTRODUCTION}

The improvement of the experimental schemes for the measurement of very weak forces, in particular, for gravitational-wave detection, has stimulated a careful examination of their ultimate precision [1]. Apart from its own interest, as far as the sensitivity of the designed interferometers improves, the quantum limits on their measurability become a matter of practical interest. So in their analysis it can be supposed that other noise sources have been eliminated and limit the discussion to the pure quantum noise.

When the quantum nature of radiation is taken into account, the first source of quantum noise to be considered is the photon noise, the photon-number fluctuations of the outgoing fields. Its magnitude can be reduced by increasing the mean power.

In an interferometric measurement, for instance, by means of a Michelson interferometer, a weak force is detected as a variation in the relative separation of the mirrors. This phase difference is transformed into an intensity signal. Therefore, if the mirrors can move, the radiation pressure produced by the reflection of light on them must be considered, the motion of the mirrors being sensitive to the fluctuations of the intensity in each arm of the interferometer. This disturbance increases with the mean power.

In the first derivations of the noise caused by these sources they were considered as separate effects [2]. The photon noise is evaluated assuming the mirrors at rest and the radiation pressure is calculated in terms of the internal intensities of the interferometer. Then the total noise is obtained as the sum of these separate contributions yielding an optimal sensitivity, the standard quantum limit (SQL), that is valid for all input states. Essentially each noise contribution is due to a quadrature of the vacuum entering the normally unused port of the interferometer. Then the SQL arises from the Heisenberg principle, assuming the uncertainties are equally distributed on both quadratures.

Unruh [3], and more recently Jaekel and Reynaud [4] using a semiclassical linear analysis, have shown that if both sources of noise are linearly superimposed the sen- sitivity can be pushed beyond the SQL with a proper choice of the input states.

A careful examination shows that the separate calculation cannot be completely satisfactory. Since the quantities actually observed are the output fields, all information about the measurement must be contained in their statistics [5]. Therefore all noise must be obtained as a photon noise. Moreover, the separate calculation assumes mutually incompatible observations from the point of view of the uncertainty principle [6].

These difficulties can be avoided if the whole interferometer with moving mirrors is considered as performing an input-output transformation on the fields. Following the model introduced by Loudon [7] we have recently developed a full quantum transformation law including the radiation pressure for pure monochromatic fields [8]. However, in this kind of measurement, the time dependencies of the signal and noise play an important role. The detection will occur in a frequency band where the mirrors are almost free to move, while they can be considered as bounded for other frequencies. In other words, a spectral analysis is needed.

Here we present a multimode description of the interferometer action when the mirrors can move and the radiation pressure must be taken into account. In this way all the information about the measurement is carried by the output fields. The superposition previously discussed naturally emerges and all the noise is obtained as a photon-counting error. The multimode character allows the calculation of the noise spectrum. We show that this complete quantum analysis confirms the reduction of noise below the SQL.

In Sec. II we examine the field reflected by a single moving mirror and characterize its action. Then we consider the whole Michelson interferometer in Sec. III. The noise spectrum is evaluated in Sec. IV. The necessary condition for the noise reduction and the states fulfilling it are considered as well.

\section{QUANTUM THEORY OF A MOVING MIRROR}

We first analyze the simpler case of a single moving mirror illuminated by a field propagating in the $Z$ axis 
with only one polarization but all possible frequencies, that is, we shall regard the system as one dimensional. We give the transformation relating the reflected field with the incident one in terms of the mirror's motion.

An ideal mirror can be described by means of appropriate boundary conditions on the electromagnetic field. For a fixed mirror, the usual continuity requirement is the vanishing of the electric field at its position $z$

$$
E(z, t)=0 \text {. }
$$

For a moving mirror, it is natural to impose this condition in the reference frame in which it is instantaneously at rest. The electric field in this frame is [9]

$$
E^{\prime}=\frac{1}{\sqrt{1-\dot{z}^{2} / c^{2}}}\left[E(z, t)+\frac{\dot{z}}{c} B(z, t)\right],
$$

where $B$ is the magnetic field and $\dot{z}$ is the speed of the mirror. In terms of the vector potential $A$ in the Coulomb gauge, the condition $E^{\prime}=0$ is

$$
\frac{\partial A}{\partial t}+\dot{z} \frac{\partial A}{\partial z}=\frac{d}{d t} A(z(t), t)=0 .
$$

Without loss of generality we can require that

$$
A(z(t), t)=0 .
$$

Decomposing $A$ into an incoming field (propagating to the right) and an outgoing field (propagating to the left), the above equation reads

$$
A_{\text {out }}(z(t), t)=-A_{\text {in }}(z(t), t) \text {. }
$$

Expanding Eq. (2.5) in terms of Fourier components, we can express the frequency amplitudes of the outgoing field in terms of the corresponding ones for the incoming field, which completely characterize the mirror action

$$
\int \frac{d \omega}{\sqrt{\omega}} a_{\text {out }}(\omega) e^{-i \omega[t+z(t) / c]}=\int \frac{d \omega}{\sqrt{\omega}} a_{\text {in }}(\omega) e^{-i \omega[t-z(t) / c]} \text {. }
$$

The range of integration over $\omega$ in Eq. (2.6) strictly extends only from 0 to $\infty$, but it is a good approximation to take a range $-\infty$ to $\infty$ for narrow-bandwidth fields.

Since we are going to consider very weak forces, only small displacements will occur. Taking the origin of coordinates at the position of the mirror in absence of forces, we have, for the frequencies of interest

$$
\frac{\omega}{c} z(t) \ll 1
$$

With this assumption, the moving-mirror action on the field can be regarded as a small perturbation from the case of the mirror at rest. This suggests that we can expand the frequency amplitudes as a power series of the perturbation $z(t)$. This is most conveniently accomplished in terms of an iteration parameter $\lambda$ such that its powers correspond to the order of the perturbation calculation. We can then replace $\omega z(t) / c$ by $\lambda \omega z(t) / c$, and assume a solution for $a_{\text {out }}(\omega)$ in the form of a power series of $\lambda[10]$. Expanding as well the exponentials in both sides of Eq. (2.6), a recursive procedure results from equating the coefficient in the same power of the iteration parameter, providing a recursion scheme which may in principle be carried out to arbitrarily high orders. In the final results $\lambda$ is set equal to 1 . Retaining the first terms, we obtain for $a_{\text {out }}(\omega)$

$a_{\text {out }}(\omega)=a_{\text {in }}(\omega)+\frac{2 i}{c} \int d \omega^{\prime} \sqrt{\omega \omega^{\prime}} z\left(\omega-\omega^{\prime}\right) a_{\text {in }}\left(\omega^{\prime}\right)-\frac{2}{c^{2}} \int d \omega^{\prime} d \omega^{\prime \prime} \omega^{\prime} \sqrt{\omega \omega^{\prime \prime}} z\left(\omega-\omega^{\prime}\right) z\left(\omega^{\prime}-\omega^{\prime \prime}\right) a_{\text {in }}\left(\omega^{\prime \prime}\right)+\cdots$

where

$$
z(\omega)=\int d t e^{i \omega t} z(t)
$$

The same result may be obtained using the time $t^{\prime}=$ $t+\frac{z(t)}{c}$ at which we observe the output field and in this way all the dependence on $z$ is in the right-hand side of Eq. (2.6). The expansion of the exponential just gives Eq. (2.8).

These relations so far hold whether the fields are classical functions or quantum operators. To quantize the theory the frequency amplitudes become operators verifying the canonical commutation relations

$$
\begin{aligned}
& {\left[a_{\text {in }}(\omega), a_{\text {in }}^{\dagger}\left(\omega^{\prime}\right)\right]=\delta\left(\omega-\omega^{\prime}\right),} \\
& {\left[a_{\text {in }}(\omega), a_{\text {in }}\left(\omega^{\prime}\right)\right]=0 .}
\end{aligned}
$$

In the quantum case, the moving mirror can be considered as performing an input-output transformation, that can be represented by an operator $U_{z}$ such that

$$
a_{\text {out }}(\omega)=U_{z}^{\dagger} a_{\text {in }}(\omega) U_{z} .
$$

Alternatively, the mirror action becomes a transformation from the input state vector | in) into the output state $\mid$ out $\rangle=U_{z} \mid$ in $\rangle$.

The operator $U_{z}$ performing the transformation (2.8) is

$$
U_{z}=\exp \left(\frac{2 i}{c} \int d \omega d \omega^{\prime} \sqrt{\omega \omega^{\prime}} z\left(\omega-\omega^{\prime}\right) a_{\mathrm{in}}^{\dagger}(\omega) a_{\mathrm{in}}\left(\omega^{\prime}\right)\right),
$$

as can be easily checked by using the well-known relation

$$
e^{-A} B e^{A}=B+[B, A]+\frac{1}{2}[[B, A], A]+\cdots .
$$

This operator $U_{z}$ is unitary provided $z^{*}(-\Omega)=z(\Omega)$, so the commutations relations (2.10) are preserved for the output field.

Now, we focus in the case when the force acting on the mirror is due to the radiation pressure force, that can be taken as twice the momentum flow 


$$
F_{\mathrm{RP}}(t)=\frac{2 \hbar}{c} \int d \omega d \omega^{\prime} \sqrt{\omega \omega^{\prime}} e^{i\left(\omega-\omega^{\prime}\right) t} a_{\mathrm{in}}^{\dagger}(\omega) a_{\mathrm{in}}\left(\omega^{\prime}\right)
$$

Considering this flow to be mainly due to a narrow frequency band around some frequency $\omega_{0}, F_{\mathrm{RP}}$ can be approximated by

$$
F_{\mathrm{RP}}(t)=2 \hbar k_{0} a_{\text {in }}^{\dagger}(t) a_{\text {in }}(t)
$$

where $k_{0}=\omega_{0} / c$ and the operator $a_{\text {in }}(t)$ is

$$
a_{\text {in }}(t)=\int d \omega e^{-i \omega t} a_{\text {in }}(\omega) .
$$

The commutation relations in the time domain derived from (2.10) are

$$
\begin{aligned}
& {\left[a_{\text {in }}(t), a_{\text {in }}^{\dagger}\left(t^{\prime}\right)\right]=\delta\left(t-t^{\prime}\right),} \\
& {\left[a_{\text {in }}(t), a_{\text {in }}\left(t^{\prime}\right)\right]=0 .}
\end{aligned}
$$

With this definition the operator $n_{\text {in }}(t)=a_{\text {in }}^{\dagger}(t) a_{\text {in }}(t)$ can be interpreted as the flux of the light beam in units of photons per unit time.

Let us characterize the response of the mirror to the force $F_{\mathrm{RP}}(t)$ by the linear susceptibility $\chi[11]$

$$
z(t)=\int d t^{\prime} \chi\left(t-t^{\prime}\right) F_{\mathrm{RP}}\left(t^{\prime}\right)
$$

valid, in particular, for a mirror held in its equilibrium position by a damped harmonic force, in which case the susceptibility in the frequency domain reads [12]

$$
\chi(\Omega)=\frac{1}{M\left(\Omega_{M}^{2}-\Omega^{2}-i \Gamma \Omega\right)},
$$

where $\Omega_{M}$ is the eigenfrequency of the system with mass $M$ and damping constant $\Gamma$.

Then the transformation operator including the radiation pressure is obtained simply by considering the dependence of $z(t)$ on the input power. Using the narrowband approximation $U_{z}$ can be expressed as

$$
U_{z}=\exp \left(i 2 k_{0} \int d t z(t) a_{\mathrm{in}}^{\dagger}(t) a_{\mathrm{in}}(t)\right) .
$$

Taking into account the time dependence of $z(t)$ in Eq. (2.18), the final expression for the transformation is

$$
U_{z}=\exp \left(i 4 \hbar k_{0}^{2} \int d t d t^{\prime} \chi\left(t-t^{\prime}\right) n_{\mathrm{in}}(t) n_{\mathrm{in}}\left(t^{\prime}\right)\right),
$$

the unitarity of the operator being preserved after this substitution [13]. In the next section we shall consider the whole interferometer with this mirror action.

\section{THE MICHELSON INTERFEROMETER WITH MOVING MIRRORS}

In this section we derive the input-output transformation law for the Michelson interferometer schematized in Fig. 1 when mirrors can move in order to detect a small

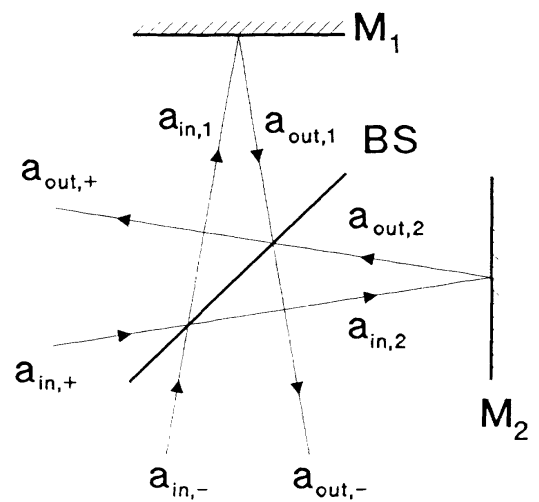

FIG. 1. Scheme of the Michelson interferometer showing the input, output, and internal modes.

force. For the modes propagating in each arm, the situation is essentially equivalent to the problem we have discussed in Sec. II, so that model can be applied here.

The interferometer can be considered as consisting of two input ports (in, + ), usually illuminated by a laser beam, and (in,-), normally unused; two output ports (out, + ) and (out, - ) and two internal paths 1 and 2 with path lengths in darkness $L_{1}$ and $L_{2}$.

The lossless beam splitter couples the internal modes in the interferometer to the input (output) modes. The relation between the input-mode annihilation operators is (see Fig. 1)

$$
\left(\begin{array}{c}
a_{\mathrm{in}, 1} \\
a_{\mathrm{in}, 2}
\end{array}\right)=\left(\begin{array}{c}
r_{+} t_{-} \\
t_{+} r_{-}
\end{array}\right)\left(\begin{array}{c}
a_{\mathrm{in},+} \\
a_{\mathrm{in},-}
\end{array}\right),
$$

where the conditions for a unitary coupling matrix are

$$
\begin{gathered}
\left|r_{+}\right|^{2}+\left|t_{-}\right|^{2}=\left|r_{-}\right|^{2}+\left|t_{+}\right|^{2}=1, \\
\delta_{r_{+}}-\delta_{t_{+}}=\delta_{t_{-}}-\delta_{r_{-}}+\pi .
\end{gathered}
$$

As we shall see later, in order to optimize the response of the interferometer the choice

$$
\begin{gathered}
\left|r_{-}\right|=\left|t_{-}\right|=\left|r_{+}\right|=\left|t_{+}\right|=\frac{1}{\sqrt{2}} \\
\delta_{r_{-}}=\delta_{t_{+}}=0, \quad \delta_{r_{+}}=-\delta_{t_{-}}=\frac{\pi}{2},
\end{gathered}
$$

and $L_{1}=L_{2} \equiv L_{0}$ is especially convenient.

The annihilation operators for the output fields are related to the corresponding ones for the input fields via a unitary transformation $U$

$$
a_{\text {out }, \mathrm{j}}(\omega)=U^{\dagger} a_{\mathrm{in}, \mathrm{j}}(\omega) U,
$$

where $j=+,-$. This transformation can be obtained by suitably combining the unitary operators representing the action of each one of the elements of the interferometer in the form

$$
U=U_{\mathrm{BS}} U_{0} U_{z} U_{0} U_{\mathrm{BS}} .
$$

Here $U_{\mathrm{BS}}$ is the unitary transformation for the beam splitter, which can be easily obtained from Eq. (3.1) [14]; 
$U_{0}$ represents the free propagation

$U_{0}=\exp \left(\frac{i}{c} \int d \omega \omega\left[L_{1} n_{\mathrm{in}, 1}(\omega)+L_{2} n_{\mathrm{in}, 2}(\omega)\right]\right)$,

and $U_{z}$ is the moving-mirror action we have discussed in the preceding section, which, for identical mirrors, can be written as

$$
\begin{array}{r}
U_{z}=\exp \left(i 4 \hbar k _ { 0 } ^ { 2 } \int d t d t ^ { \prime } \chi ( t - t ^ { \prime } ) \left[n_{\mathrm{in}, 1}(t) n_{\mathrm{in}, 1}\left(t^{\prime}\right)\right.\right. \\
\left.\left.+n_{\mathrm{in}, 2}(t) n_{\mathrm{in}, 2}\left(t^{\prime}\right)\right]\right) .
\end{array}
$$

This total transformation enables one to know the output fields in terms of the input ones, and therefore the result of any measure on them.

We assume that the detectors, placed at the two output channels of the interferometer, give a response proportional to the output photon flows. This assumption is equivalent to a detection of energy flow taking the narrow-band approximation [15]. The optimum response to small displacements of the mirrors can be reached either by phase-modulation techniques or balanced detection, both schemes giving similar sensitivities, at least for ideal conditions [16]. When balanced detection is used the difference between the two output flows is measured around a working point where it is zero. The operator of interest in this case is

$$
\begin{aligned}
n_{B}(t) & =n_{\text {out },+}(t)-n_{\text {out },-}(t) \\
& =U^{\dagger}\left[n_{\text {in },+}(t)-n_{\text {in, }-}(t)\right] U .
\end{aligned}
$$

With the knowledge of the transformation operator for the interferometer, we can express the detection operator (3.8) in terms of the operators of the input fields including the effect of the radiation pressure, getting [5]

$$
\begin{aligned}
n_{B}(t)= & a_{\mathrm{in},-}^{\dagger}(\tau) \sin [\Lambda(\tau)] a_{\mathrm{in},-}(\tau) \\
& -a_{\mathrm{in},+}^{\dagger}(\tau) \sin [\Lambda(\tau)] a_{\mathrm{in},+}(\tau) \\
& +i\left\{a_{\mathrm{in},+}^{\dagger}(\tau) \cos [\Lambda(\tau)] a_{\mathrm{in},-}(\tau)\right. \\
& \left.\quad-a_{\mathrm{in},-}^{\dagger}(\tau) \cos [\Lambda(\tau)] a_{\mathrm{in},+}(\tau)\right\}
\end{aligned}
$$

where

$$
\begin{aligned}
\Lambda(\tau)=8 \hbar k_{0}^{2} \int d t^{\prime} \chi_{R}\left(\tau-t^{\prime}\right) & \\
\times & {\left[a_{\mathrm{in},-}^{\dagger}\left(t^{\prime}\right) a_{\mathrm{in},+}\left(t^{\prime}\right)\right.} \\
& \left.+a_{\mathrm{in},+}^{\dagger}\left(t^{\prime}\right) a_{\mathrm{in},-}\left(t^{\prime}\right)\right],
\end{aligned}
$$

with $\chi_{R}\left(t-t^{\prime}\right)=\frac{1}{2}\left[\chi\left(t-t^{\prime}\right)+\chi\left(t^{\prime}-t\right)\right]$ and $\tau=t-$ $2 L_{0} / c$. Since the radiation pressure effects are small according to Eq. (2.7), we can expand $n_{B}(t)$ up to second powers in $\Lambda$, obtaining

$$
\begin{aligned}
n_{B}(t)= & i\left[a_{\mathrm{in},+}^{\dagger}(\tau) a_{\mathrm{in},-}(\tau)-a_{\mathrm{in},-}^{\dagger}(\tau) a_{\mathrm{in},+}(\tau)\right] \\
& +a_{\mathrm{in},-}^{\dagger}(\tau) \Lambda(\tau) a_{\mathrm{in},-}(\tau)-a_{\mathrm{in},+}^{\dagger}(\tau) \Lambda(\tau) a_{\mathrm{in},+}(\tau) \\
+ & +\frac{i}{2}\left[a_{\mathrm{in},-}^{\dagger}(\tau) \Lambda^{2}(\tau) a_{\mathrm{in},+}(\tau)\right. \\
& \left.\quad-a_{\mathrm{in},+}^{\dagger}(\tau) \Lambda^{2}(\tau) a_{\mathrm{in},-}(\tau)\right]
\end{aligned}
$$

The first term in this equation is just the detection operator in absence of radiation pressure, while the other terms are successive corrections. This relation for the detection operator allows us to obtain all the information about the measurement once we specify the states entering the interferometer.

\section{NOISE SPECTRUM}

In the kind of measurements we are discussing, the input port $($ in,+$)$ of the interferometer is illuminated by a laser beam, so for this channel we consider a coherent state in a single mode of frequency $\omega_{0}[17]$,

$$
\begin{aligned}
& \left.\left.a_{\text {in, }+}(\omega) \mid \text { in },+\right\rangle=\alpha \delta\left(\omega-\omega_{0}\right) \mid \text { in },+\right\rangle, \\
& \left.\left.a_{\text {in, }+}(t) \mid \text { in, }+\right\rangle=\alpha e^{-i \omega_{0} t} \mid \text { in },+\right\rangle .
\end{aligned}
$$

Before evaluating the noise for a particular choice of the input state in channel (in,-) we can see its features better if we consider the coherent intensity to be much higher than the corresponding one in the (in,-), as is often the case. So, it will be a good approximation to replace the operator $a_{\text {in, }}(t)$ by the complex amplitude $\alpha e^{-i \omega_{0} t}$ neglecting its fluctuations. Therefore we can take

$n_{B}(t) \approx-\sqrt{f}\left[Y(\tau)+8 \hbar k_{0}^{2} f \int d t^{\prime} \chi_{R}\left(\tau-t^{\prime}\right) X\left(t^{\prime}\right)\right]$

where $f=|\alpha|^{2}$ represents the flux in units of photons per unit time and

$$
\begin{aligned}
& X(t)=e^{i \omega_{0} t} a_{\mathrm{in},-}(t)+e^{-i \omega_{0} t} a_{\mathrm{in},-}^{\dagger}(t), \\
& Y(t)=i\left[e^{-i \omega_{0} t} a_{\mathrm{in},-}^{\dagger}(t)-e^{i \omega_{0} t} a_{\mathrm{in},-}(t)\right]
\end{aligned}
$$

are the quadratures of the (in,-) field.

In a separate calculation each noise source can be assigned to the fluctuations of one quadrature of the (in,-) field. The use of squeezed states allows an adequate balance between them to reach the SQL

Since only photon noise can be measured, we have argued before that a superposition of both sources of noise should appear in the detection. We see in Eq. (4.2) that this is the case in this unified calculation, being the key to overcome the SQL. In fact, in the fluctuations of $n_{B}(t)$ the correlations between the two quadratures of the (in,-) field contribute to the noise, a fact absent in any separate calculation. The necessary condition to the reduction of noise is that the state (in,-) presents the 
appropriate correlation. This can be done, for instance, with the use of a correlated squeezed vacuum. Therefore we consider the mode (in,-) to be obtained from the output of a degenerate parametric amplifier in the case of a single-ended cavity, which has proved to be one of the most successful ways to achieve it [18]. In such a case we have [19]

$$
\begin{aligned}
\left\langle a_{\mathrm{in},-}(\omega)\right\rangle & =0 \\
\left\langle a_{\mathrm{in},-}^{\dagger}(\omega) a_{\mathrm{in},-}\left(\omega^{\prime}\right)\right\rangle & =N(\omega) \delta\left(\omega-\omega^{\prime}\right), \\
\left\langle a_{\mathrm{in},-}(\omega) a_{\mathrm{in},-}\left(\omega^{\prime}\right)\right\rangle & =M(\omega) \delta\left(2 \omega_{0}-\omega-\omega^{\prime}\right),
\end{aligned}
$$

$$
\begin{aligned}
M(\omega)=\frac{\epsilon \gamma}{2} e^{i \theta}[ & \frac{1}{\left(\frac{\gamma}{2}-\epsilon\right)^{2}+\left(\omega_{0}-\omega\right)^{2}} \\
& \left.+\frac{1}{\left(\frac{\gamma}{2}+\epsilon\right)^{2}+\left(\omega_{0}-\omega\right)^{2}}\right],
\end{aligned}
$$

where $\epsilon$ and $\theta$ are the modulus and phase of the amplification constant, $\gamma$ is the cavity damping, and we have assumed that the frequency around which the squeezing has been produced coincides with the frequency of the coherent input.

The role of the interferometer is to transform a small variation of the relative separation of the mirrors $z=$ $L_{1}-L_{2}$ into a variation in the output photon flows. Under the conditions assumed it is easy to see that if there are no external forces acting on the mirrors

$$
\left\langle n_{B}(t)\right\rangle=0,
$$

while a small variation of $z$ gives a signal

$$
\left\langle n_{B}(t)\right\rangle=2 k_{0} f z
$$

so the interferometer is effectively prepared at the optimum working point.

The fluctuations in the output photon flows cause the corresponding uncertainties in the relative separation of the mirrors. We next calculate the spectrum of the output if there are no external forces acting on the mirrors. That is to say, the background noise spectrum from which the signal should be detected.

The incident states we have chosen allows us to consider $n_{B}(t)$ as a stationary variable. Therefore the spectrum of fluctuations can be obtained as the Fourier transform

$$
S_{n n}(\Omega)=\int d t^{\prime} e^{i \Omega t^{\prime}}\left\langle\Delta n_{B}\left(t+t^{\prime}\right) \Delta n_{B}(t)\right\rangle,
$$

where

$$
\Delta n_{B}(t)=n_{B}(t)-\left\langle n_{B}(t)\right\rangle
$$

that in our case becomes

$$
S_{n n}(\Omega)=\int d t^{\prime} e^{i \Omega t^{\prime}}\left\langle n_{B}\left(t+t^{\prime}\right) n_{B}(t)\right\rangle .
$$

It should be noted that there is no ordering problem with the operators because $\left[n_{B}(t), n_{B}\left(t^{\prime}\right)\right]=0$.

Since we have assumed the radiation pressure to be a small effect [see Eq. (2.7)] and the coherent intensity to be much higher that the squeezed one, we can use the expansion (3.11), retaining just the greatest term in each power of $\chi$. With all these in mind the noise spectrum is

$$
\begin{aligned}
S_{n n}(\Omega)= & f S_{Y Y}(\Omega)+16 \hbar k_{0}^{2} f^{2} \chi_{R}(\Omega) S_{X Y}(\Omega) \\
& +64 \hbar^{2} k_{0}^{4} f^{3} \chi_{R}^{2}(\Omega) S_{X X}(\Omega),
\end{aligned}
$$

where

$$
\begin{aligned}
S_{X X}(\Omega)= & 1+N\left(\omega_{0}+\Omega\right)+N\left(\omega_{0}-\Omega\right) \\
& +M\left(\omega_{0}+\Omega\right)+M^{*}\left(\omega_{0}-\Omega\right), \\
S_{Y Y}(\Omega)= & 1+N\left(\omega_{0}+\Omega\right)+N\left(\omega_{0}-\Omega\right) \\
& -M\left(\omega_{0}+\Omega\right)-M^{*}\left(\omega_{0}-\Omega\right), \\
S_{X Y}(\Omega)= & i\left[M^{*}\left(\omega_{0}-\Omega\right)-M\left(\omega_{0}+\Omega\right)\right] .
\end{aligned}
$$

These last functions are nothing but the correlation functions of the operators

$$
\begin{aligned}
X(\Omega) & =\int d t e^{i \Omega t} X(t)=a_{\mathrm{in},-}\left(\omega_{0}+\Omega\right)+a_{\mathrm{in},-}^{\dagger}\left(\omega_{0}-\Omega\right) \\
Y(\Omega) & =\int d t e^{i \Omega t} Y(t) \\
& =i\left[a_{\mathrm{in},-}^{\dagger}\left(\omega_{0}-\Omega\right)-a_{\mathrm{in},-}\left(\omega_{0}+\Omega\right)\right]
\end{aligned}
$$

that is

$$
\begin{aligned}
& S_{X X}(\Omega) \delta\left(\Omega+\Omega^{\prime}\right)=\left\langle X(\Omega) X\left(\Omega^{\prime}\right)\right\rangle \\
& S_{Y Y}(\Omega) \delta\left(\Omega+\Omega^{\prime}\right)=\left\langle Y(\Omega) Y\left(\Omega^{\prime}\right)\right\rangle \\
& S_{X Y}(\Omega) \delta\left(\Omega+\Omega^{\prime}\right)=\frac{1}{2}\left\langle X(\Omega) Y\left(\Omega^{\prime}\right)+Y(\Omega) X\left(\Omega^{\prime}\right)\right\rangle
\end{aligned}
$$

We have not yet completely specified the detector response to the photon flow. The detector can be characterized by a response function $\eta$ in such a way that the detected output is

$$
N_{B}(t)=\int d t^{\prime} \eta\left(t-t^{\prime}\right) n_{B}\left(t^{\prime}\right)
$$

The function $\eta$ contains the frequency response as well as the duration of the measure. In the frequency domain its effect will appear as a multiplicative factor as

$$
S_{N N}(\Omega)=|\eta(\Omega)|^{2} S_{n n}(\Omega) .
$$

We can consider this frequency-dependent response of the detector as a filter selecting the appropriate frequency band centered at the signal frequency.

The previous fluctuations in the detected output photon flows cause the final indetermination in $z$ to be 


$$
\left\langle(\Delta z)^{2}\right\rangle=\frac{B}{2 k_{0}^{2} f^{2}} \overline{S_{n n}},
$$

where the bar denotes the mean value over the normalized frequency distribution induced by the filtering detector in Eq. (4.16), and $B$ its bandwidth

$$
B=\frac{1}{2} \int d \Omega|\eta(\Omega)|^{2}
$$

Using now Eq. (4.11) for $S_{n n}$, we have

$$
\begin{aligned}
\left\langle(\Delta z)^{2}\right\rangle=2 B[ & \frac{1}{4 k_{0}^{2} f} \overline{S_{Y Y}}+4 \hbar \overline{\chi_{R} S_{X Y}} \\
& \left.+16 \hbar^{2} k_{0}^{2} f \overline{\chi_{R}^{2} S_{X X}}\right] .
\end{aligned}
$$

Actually with the present available sources of squeezed states the spectrum of fluctuations of the output is much broader than the detection bandwidth of this kind of measurements [20]. Then we can consider the whitenoise limit of infinite-band squeezed vacuum, neglecting the frequency dependence in Eq. (4.5), and consider the correlations in Eq. (4.12) to be independent of the frequency, verifying the relation

$$
S_{X X} S_{Y Y}=1+S_{X Y}^{2}
$$

In these conditions, when $f$ is varied, the minimum of Eq. (4.19) gives

$$
\left\langle(\Delta z)^{2}\right\rangle=8 B \hbar\left(\sqrt{1+S_{X Y}^{2}} \sqrt{\overline{\chi_{R}^{2}}}+S_{X Y} \overline{\chi_{R}}\right),
$$

for the optimum photon flow

$$
f_{\min }=\frac{1}{8 \hbar k_{0}^{2} \sqrt{\overline{\chi_{R}^{2}}}} \sqrt{\frac{S_{Y Y}}{S_{X X}}} .
$$

When the field entering the channel (in,-) is the vacuum $\left(S_{X X}=S_{Y Y}=1, S_{X Y}=0\right)$, the minimum uncertainty gives the $\mathrm{SQL}$

$$
\left\langle(\Delta z)_{\mathrm{SQL}}^{2}\right\rangle=8 B \hbar \sqrt{\overline{\chi_{R}^{2}}}
$$

and the optimum photon flow in this case

$$
f_{\mathrm{SQL}}=\frac{1}{8 \hbar k_{0}^{2} \sqrt{\overline{\chi_{R}^{2}}}}
$$

translates to an unpractically high optimum power. Caves's proposal [2] corresponds to squeezing the $Y$ quadrature of the (in,-) field, which leads to the same SQL uncertainty, but for a smaller input power.

As we have mentioned, the unified calculation shows the superposition of both sources of fluctuations, ex- pressed in Eq. (4.2), that leads to the uncertainty depending upon the correlations between $X$ and $Y$ variables. This fact can be used to reduce the noise in front of the uncorrelated case. Essentially, the fluctuations of the (in,-) field must be squeezed in the appropriate direction of the $X Y$ plane.

Now $S_{X Y}$ can be varied giving a minimum uncertainty

$$
\left\langle(\Delta z)^{2}\right\rangle=8 B \hbar \sqrt{\overline{\bar{\chi}_{R}^{2}}-{\overline{\chi_{R}}}^{2}},
$$

when

$$
S_{X Y}=-\frac{\overline{\chi_{R}}}{\sqrt{\overline{\chi_{R}^{2}}-{\overline{\chi_{R}}}^{2}}} .
$$

This lower bound is clearly below the SQL in Eq. (4.23). The precise amount of noise reduction and the feasibility of achieving it, depends both on the filtering distribution and the susceptibility. A lower bound for the sensitivity has been discussed by Jaekel and Reynaud [4] assuming that noise can be optimized at each frequency. This analysis is formally equivalent to the noise reduction in a single-mode model [8].

\section{CONCLUSIONS}

The quantities actually observed in interferometric measurements are the output fields. So the discussion about the quantum noise should be done in terms of their statistics. In this work, we have presented a multimode quantum model for the Michelson interferometer with moving mirrors, giving a unitary transformation relating the output fields with the input ones that includes the radiation pressure effect. With a multimode description the noise spectrum can be evaluated. That would in particular allow one to discriminate a signal from the background noise. We have shown that, as it could be expected, photon noise and radiation pressure are not independent sources of uncertainty. Our analysis supports previous results, appearing in the total noise a correlation term, can be used, with a judicious choice of the input states, to push the uncertainty below the SQL.

The interferometer described here is, of course, a highly idealized system. Real interferometers are subject to other error sources that must be overcome in the design of real experiments and render the detection of gravitational waves a formidable experimental task. However, as interferometers are made longer, the ultimate quantum limits on their measurability become a matter of practical interest.

\section{ACKNOWLEDGMENT}

We are much indebted to Professor E. Bernabeu for his continual advice and interest in the present work.
[1] H. P. Yuen, Phys. Rev. Lett. 51, 719 (1983); M. Ozawa, ibid. 60, 385 (1988); Phys. Rev. A 41, 1735 (1990); W. T. Ni, ibid. 33, 2225 (1986); R. S. Bondurant and J. H. Shapiro, Phys. Rev. D 30, 2548 (1984).
[2] C. M. Caves, Phys. Rev. Lett. 45, 75 (1980); Phys. Rev. D 23, 1693 (1981).

[3] W. G. Unruh, in Quantum Optics, Experimental Gravitation and Measurement Theory, edited by P. Meystre 
and M. O. Scully (Plenum, New York, 1983), p. 647.

[4] M. T. Jaekel and S. Reynaud, Europhys. Lett. 13, 301 (1990).

[5] A. Luis and L. L. Sánchez-Soto, J. Mod. Opt. 38, 971 (1991).

[6] B. Marx, Nature 287, 276 (1980).

[7] R. Loudon, Phys. Rev. Lett. 47, 815 (1981).

[8] A. Luis and L. L. Sánchez-Soto, Opt. Commun. (to be published).

[9] S. Sarkar, J. Phys. A 21, 971 (1988); G. T. Moore, J. Math. Phys. 11, 2679 (1970)

[10] L. Schiff, Quantum Mechaniccs (McGraw-Hill, New York, 1949), Chap. VII; G. Dattoli, J. C. Gallardo, and A. Torre, An Algebraic View to the Operatorial Ordering and its Applications to Optics [Riv. Nuovo Cimento 11, 1 (1988)].

[11] P. Martin, in Many Body Physics, Les Houches Lectures, edited by C. de Witt and R. Balian (Gordon and Breach, New York, 1968), p. 39.
[12] A. Brillet, Ann. Phys. (Paris) 10, 219 (1985).

[13] A. Luis and L. L. Sánchez-Soto, J. Phys. A 24, 2083 (1991).

[14] B. Yurke, S. L. McCall, and J. R. Klauder, Phys. Rev. A 33, 4033 (1986).

[15] R. S. Bondurant, Phys. Rev. A 32, 2797 (1985).

[16] J. Gea-Banacloche and G. Leuchs, J. Opt. Soc. Am. B 4, 1667 (1987); J. Mod. Opt. 34, 793 (1987); 36, 1277 (1989).

[17] K. J. Blow, R. Loudon, S. J. D. Phoenix, and T. J. Shepherd, Phys. Rev. A 42, 4102 (1990)

[18] L. Wu, H. J. Kimble, J. L. Hall, and H. Wu, Phys. Rev. Lett. 57, 2520 (1986)

[19] M. J. Collet and C. W. Gardiner, Phys. Rev. A 30, 1386 (1984).

[20] D. Schoemaker, R. Schilling, L. Schnupp, W. Winkler, K. Maischberger, and A. Rüdiger, Phys. Rev. D 38, 423 (1988). 